\title{
Fail-Safe Design by Outer Cover of High Pressure Vessel for Food Processing
}

\author{
Yuichi Otsuka' ${ }^{1}$ Jyunpei Fujii' ${ }^{2}$, Masaki Takato ${ }^{2}$, Yoshiharu Mutoh ${ }^{3}$ \\ ${ }^{1}$ Top Runner Incubation Center for Academia-Industry Fusion, Nagaoka University \\ of Technology, Niigata, Japan \\ ${ }^{2}$ Department of System Safety, Graduate School of Management and Technology, Nagaoka \\ University of Technology, Niigata, Japan \\ ${ }^{3}$ Department of System Safety, Nagaoka University of Technology, Niigata, Japan \\ E-mail:otsuka@vos.nagaokaut.ac.jp \\ Received September 26, 2011; revised November 2, 2011; accepted November 10, 2011
}

\begin{abstract}
This paper demonstrates the effectiveness of outer-cover of pressure vessel in order to prevent risk scenario by brittle fracture. The Fail-Safe design introducing the concept of a standby redundant system is applied to the high-pressure vessel so that the safety of the operator can be assured in case of the vessel fractures. Based on the limit state design (LSD) concept, a fracture mode is predicted as plastic collapse and brittle fracture. Dimensions of a cover are determined by considering failure modes of plastic collapse and brittle fracture. Failure experiment with pre-cracked vessel can clearly show the effectiveness of the proposed vessel for fail-safe design.
\end{abstract}

Keywords: Food Processing, Pressure Vessel, Fail-Safe Design, Safety Engineering

\section{Introduction}

It is reported in a previous study [1] that the high pressure processing of foods will produce a variety of advantageous effects such as enhancement of sterilization force and gustatory sense, and condensation of an "umami” (good) flavor. In this study, light and safe highpressure processing equipment is being developed, assuming that the equipment will be used in small-to-medium businesses. In most conventional high-pressure processing equipment, high pressure is created directly with such devices as a piston but they have a deficiency in that the size of the entire equipment tends to be large. In order to mitigate this deficiency, indirect pressurization and sealed systems are proposed [2] as small and light high-pressure processing systems. In the indirect pressurization system, high pressure is created with the help of a pressure intensifier; in the sealed system, a locking bar type pin, such as the one shown in Figures $\mathbf{1}$ and 2, is used. With the adoption of this sealed system, the high-pressure vessel can be light. Since the load is supported at the corners on the locking bar guide (or lever guide in Figure 2), however, the stress will be concentrated on the corners, possibly resulting in rapid fracture in the early phase of operations. To resolve this stress concentration issue, we optimized, from the strength reliability point of view, the shape of the seal section in order to mitigate the concentration. However, it is impossible, only from the reliability point of view, to rationally derive countermeasures to assure the safety of the operator against fracture of the equipment.

In this study, the Fail-Safe design introducing the concept of a standby redundant system [3] is applied to the high-pressure vessel so that the safety of the operator can be assured in case of the vessel fractures, and the intrinsic safety feature can be implemented in the equipment. Based on the limit state design (LSD) concept, a fracture mode is predicted, and countermeasures against the fracture mode are investigated. From the results of the investigation, fracture testing of the pre-cracked high-pressure vessel is carried out and the resulting effectiveness of the concept is confirmed and reported in this paper.

\section{Fail-Safe Design by Outer Cover of Pressure Vessel}

\subsection{Installation of Outer Cover}

As illustrated in Figure 3(a), an outer cover is installed on the outside of the pressure vessel. The cover bears no 


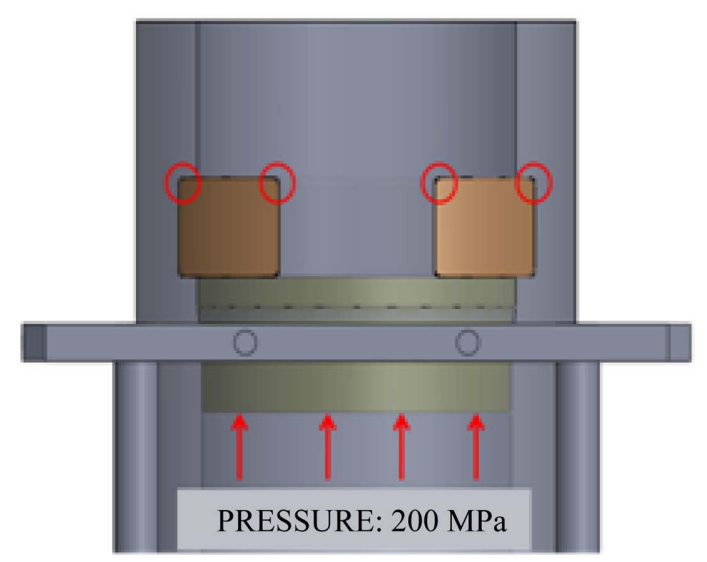

Figure 1. Pin-arm sealed pressure vessel structure for food processing.

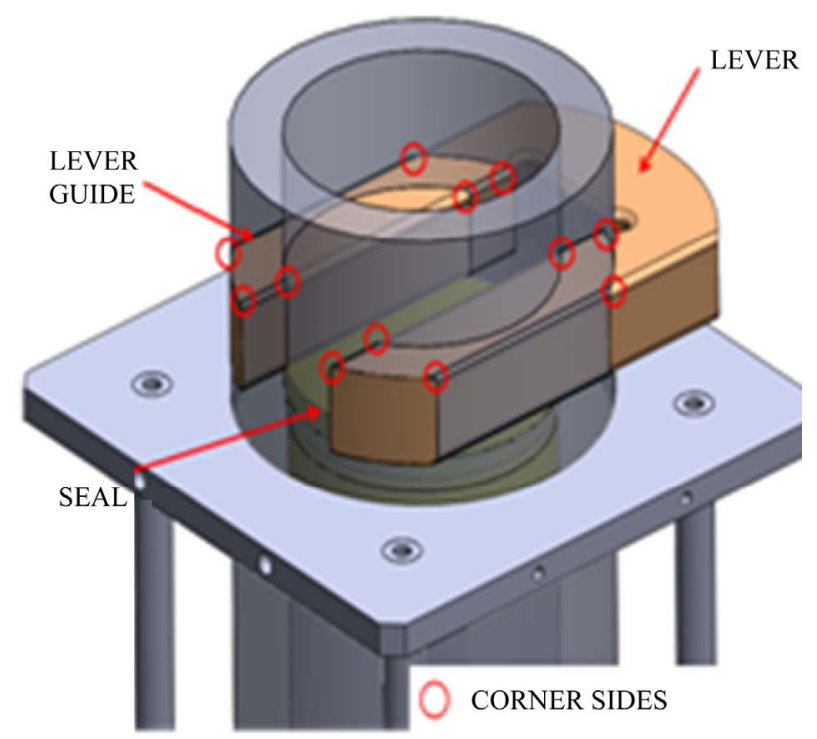

Figure 2. Upper view of pressure vessel.

load as long as the equipment is operating normally. Only in case of fracture of the locking bar guide (lever guide), it bears the load and prevents fragments of the fractured guide from being scattered. This cover can be regarded as a sort of standby redundant system because it does not function under the normal operation conditions. In the following sections, the structure of the cover preventing fragments from being scattered is studied in terms of avoiding plastic collapse and rapid fracture originating from the stress concentration area.

\subsection{Calculation of Penetration Thickness Caused by Plastic Collapse}

As illustrated in Figure 3(b), the internal energy of highpressure liquid is converted into the dynamic energy of objects generated by and scattered from the locking bar
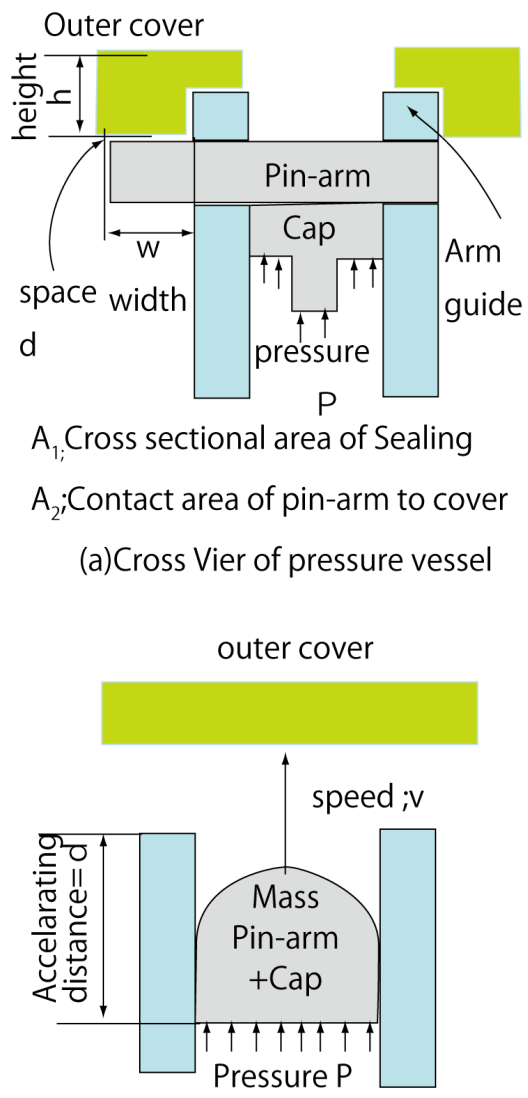

(b)Approximation model of contact

Figure 3. Bullet model of fracture of protective cover.

guide fracture. The following equation is used to calculate the magnitude of energy that causes the plastic deformation.

$$
h=P A_{1} d / \sigma_{B} A_{2}
$$

The following figures are entered into the Equation (1) to calculate the energy: $\alpha$ : Correction factor for energy conversion efficiency ( $\alpha=1$ assuming the worst case), $P$ : Internal pressure $\left(P=2.0 \times 10^{8}\left[\mathrm{~N} / \mathrm{m}^{2}\right]\right) \mathrm{m}$ : Sum of arm mass and cover mass ( $m=14.2[\mathrm{~kg}]), A_{1}$ : Cross sectional area of cover $\left(A_{1}=0.01606\left[\mathrm{~m}^{2}\right]\right) A_{2}$ : Contact area of arm and cover $\left(A_{2}=\right.$ See Figure $\left.4\left[\mathrm{~m}^{2}\right]\right)$, and $\sigma_{B}$ : Tensile strength of SCM435 $\left(\sigma_{B}=9.32 \times 10^{8}\left[\mathrm{~N} / \mathrm{m}^{2}\right]\right)$. The calculated penetration thickness $h$ is listed in Table $\mathbf{1}$ as a function of width $w$ and space $d$.

Here we use the following equation to estimate whether impact energy can be absorbed while cracks caused by the impact in the maximum stress concentration area are being propagated.

$$
\frac{w}{d}=\frac{P \times A_{1} \times 10^{5}}{8 v E \times l}
$$

where, $v E$ is the Charpy impact value $\left(v E=200\left[\mathrm{~J} / \mathrm{cm}^{2}\right]\right)$ 


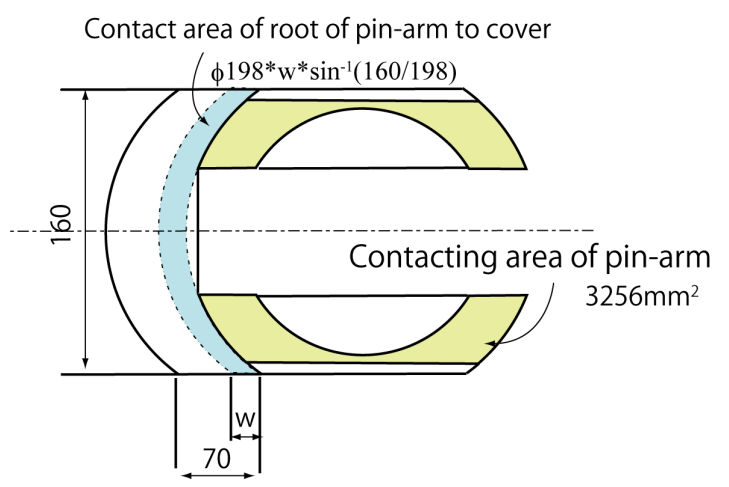

Figure 4. Definition of contacting area $A_{2}$.

Table 1. Calculated thickness $h[\mathrm{~mm}]$ by Equation (1) when the failure mode "plastic collapse" occurs from the $R$ corner of arm guide (red circle of Figure 1).

\begin{tabular}{cccccc}
\hline & \multicolumn{5}{c}{ Space $d[\mathrm{~mm}]$} \\
\cline { 2 - 4 } Width $w[\mathrm{~mm}]$ & 0.1 & 0.2 & $\underline{\mathbf{1}}$ & 2 & 5 \\
\hline 1 & 1.9 & 3.7 & 18.5 & 37 & 92.5 \\
5 & 0.4 & 0.7 & 2.6 & 7.4 & 18.5 \\
10 & 0.23 & 0.4 & 1.9 & 3.7 & 9.3 \\
15 & 0.1 & 0.2 & 1.2 & 2.5 & 6.2 \\
$\underline{\mathbf{2 0}}$ & $<0.1$ & 0.2 & $\underline{\mathbf{0 . 9}}$ & 1.9 & 4.6 \\
\hline
\end{tabular}

and $l$ is the length of the crack formed near the maximum stress concentration area. The coefficient 4 is used when the impact is applied only on one end (root) of the arm, and the coefficient is 8 when the impact is applied on both ends of an arm (i.e., on 8 locations (= 4 locations/ end $\times 2$ ends)). The calculated width ( $w$ ) is listed in Table 2 as a function of the space $(d)$ and length $(l)$ of the formed crack.

Dimensions are determined by the following process.

1) From the fracture toughness value $K_{I C}$ and maximum stress value at the $\mathrm{R}$ corner, absorbing length $l$ should be less than $10 \mathrm{~mm}$ ( green colored columns in the Tables 1 and 2)

2) From the constraint condition that the cover never contacts to the top of inner pressure vessel at the maximum pressuring condition, space $d$ should be more than $0.9 \mathrm{~mm}$ (blue colored columns in the Tables $\mathbf{1}$ and 2).

3) From the cost effectiveness condition, minimum value in the pink colored columns in Table 2 is selected, $w$ is about $20 \mathrm{~mm}$.

4) From the cost effectiveness condition, minimum value in the yellow colored columns in Table $\mathbf{1}$ is selected, $h$ is about $0.9 \mathrm{~mm}$.

Finally selected values are emphasized by italic and
Table 2. Calculated width $w[\mathrm{~mm}]$ by Equation (2) when the failure mode "brittle fracture" occurs from the R corner of arm guide (red circle of Figure 1).

\begin{tabular}{cccccc}
\hline & \multicolumn{5}{c}{ Absorbing length $l[\mathrm{~mm}]$} \\
\cline { 2 - 6 } Space $d[\mathrm{~mm}]$ & 1 & 2 & 5 & $\underline{\mathbf{1 0}}$ & 20 \\
\hline 0.1 & 20.1 & 10 & 4 & 2 & 1 \\
0.2 & 40.2 & 20.1 & 8.3 & 4 & 2 \\
$\underline{\mathbf{1}}$ & 200.8 & 100.4 & 40.2 & $\underline{\mathbf{2 0 . 1}}$ & 10 \\
2 & 401.5 & 200.8 & 80.3 & 40.2 & 20.1 \\
5 & 1003.8 & 501.9 & 200.8 & 100.4 & 50.2 \\
\hline
\end{tabular}

underlined letters in the tables. Finally selected values are emphasized by italic and underlined letters in the tables. By considering the safety coefficient for the values of $d$ and $h, d$ should be more than $1.3 \mathrm{~mm}$ and $h$ should be more than $1 \mathrm{~mm}$.

\section{Verifications of Fail-Safe Design of Pressure Vessel by Fracture Test}

On the corners of the lever guide in both a pressure vessel with the outer cover (double-wall vessel) and a vessel without the outer cover (single-wall vessel), pre-cracks of $5 \mathrm{~mm}$ in length were machined and the pre-cracked pressure vessels were subjected to fracture testing. In the testing, the pressure applied was increased by $25 \%$ to $250 \mathrm{MPa}$. Refer to Figure 1 for the corners (R sections), each of which is marked with a circle. For safety precautions during testing, all the equipment was enclosed in a protective box.

As shown in Figures 5 and 6, fracture testing of the single-wall vessel resulted in a broken guide, the inside surface had 45 degrees of slant angle and the outside surface was flat. We calculated the stress distribution around the $\mathrm{R}$ corners in maximum pressuring by using the finite element analyses (FEA) in ref. [2]. The direction of fractured surface is almost vertical to the direction of the principle component of stress, and it can be estimated that the fracture was brittle fracture because the principal normal stress can normally cause the brittle fracture.

Figure 7 shows the arm guide contacting condition before test for a vessel with outer cover. Figure 8 shows the broken arm guide for the vessel with outer cover. Angle of cracking from the $\mathrm{R}$ corner of arm guide is decreased from 45 degree as shown in Figure 8. The decrease of angle means the arm guide and the seal is contacting the outer cover in cracking and then the force from pressure $250 \mathrm{MPa}$ is distorted into the outer cover that would result in the decrease of maximum stress value at the $\mathrm{R}$ corner. The outer cover could successfully prevent the broken arm guide from flying. 


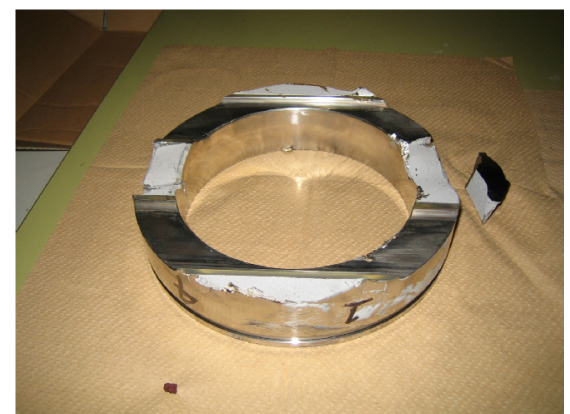

Figure 5. Flying parts of broken arm guide (The top part of Figure 1). Angle of fracture surface is about 45 degree (left and right side).

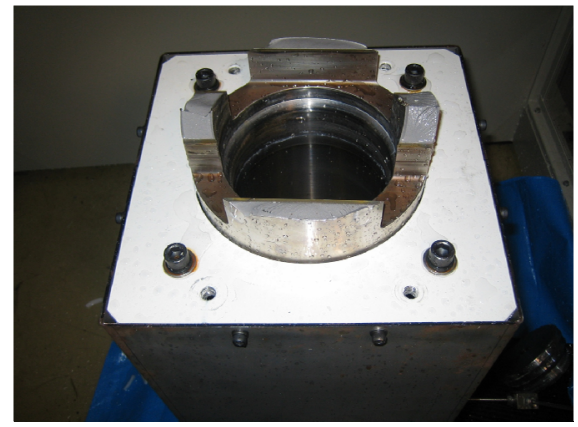

Figure 6. Remained parts of arm guide (The part shown in Figure 1). Fracture surface shows smooth brittle features.

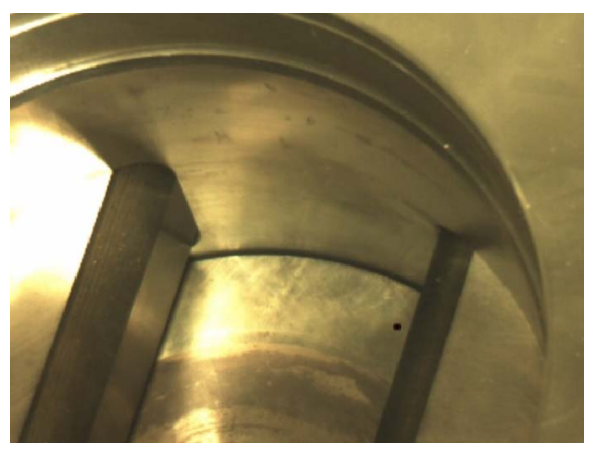

Figure 7. Arm guide contacting condition before test for a vessel with outer cover.

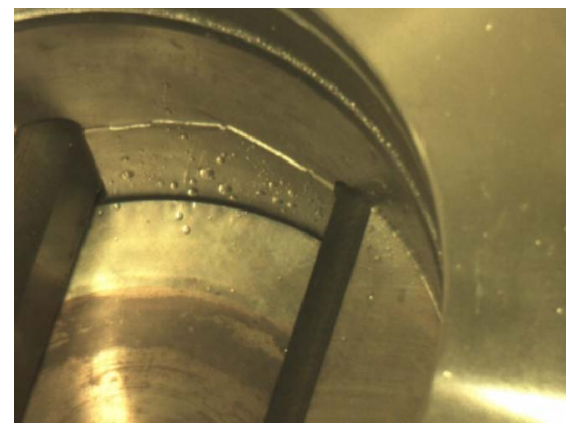

Figure 8. Broken arm guide for the vessel with outer cover. Angle of fracture surface is suppressed by the support from the outer cover during fracture.
Figure 9 shows the broken roof of the protective box by the flying parts (Figure 5) in the fracture case of the vessel without outer cover. Figure 10 shows the preserved roof of the protective box in the fracture case of the vessel with outer cover. Figure 9 can easily demonstrate the critical risk of brittle fracture which the penetrated thickness of the steel plate is $6 \mathrm{~mm}$. In the case of Figure 10, which the fracture test case for the vessel with outer cover, the plate at the roof is not damaged by the fracture and only wetted by water flow from the cracking part of the arm guide. The risk of wetting by water flow is not serious and can be acceptable for users compared with the critical damage shown in Figure 9.

Figure 11 shows change of strain during fracture propagation. In the course of measurement, fracture started when the strain signals reaches maximum (that means strain signal was lost), which implies that the fracture began from the outside. From the FEA result of the arm guide [2], maximum stress is on the inner side of the arm guide, which starting point of brittle fracture in the experiment is not corresponding. The FEA result can show that the maximum stress point moves from inner side to

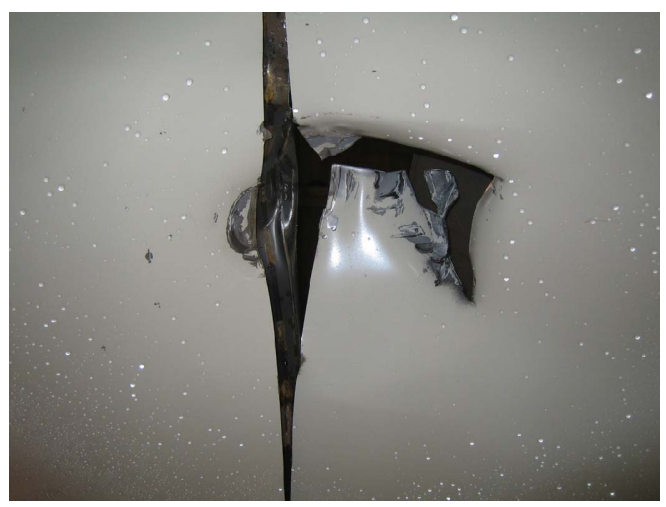

Figure 9. Broken roof of the protective box by the flying parts (Figure 5) in the fracture case of the vessel without outer cover.

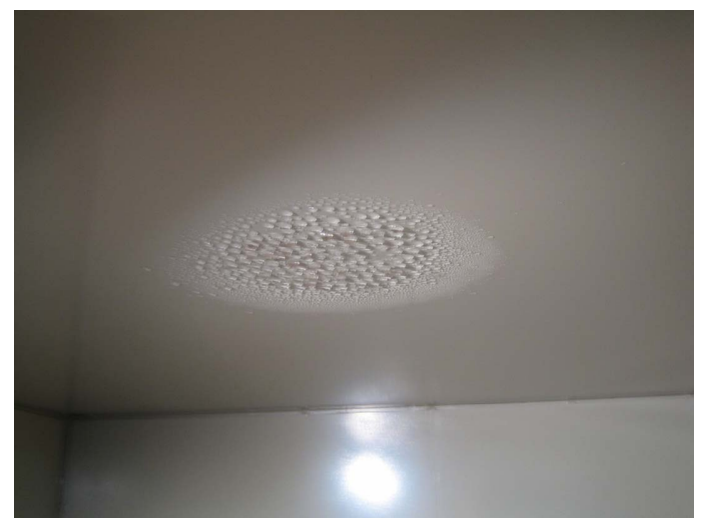

Figure 10. Preserved roof of the protective box in the fracture case of the vessel with outer cover. 


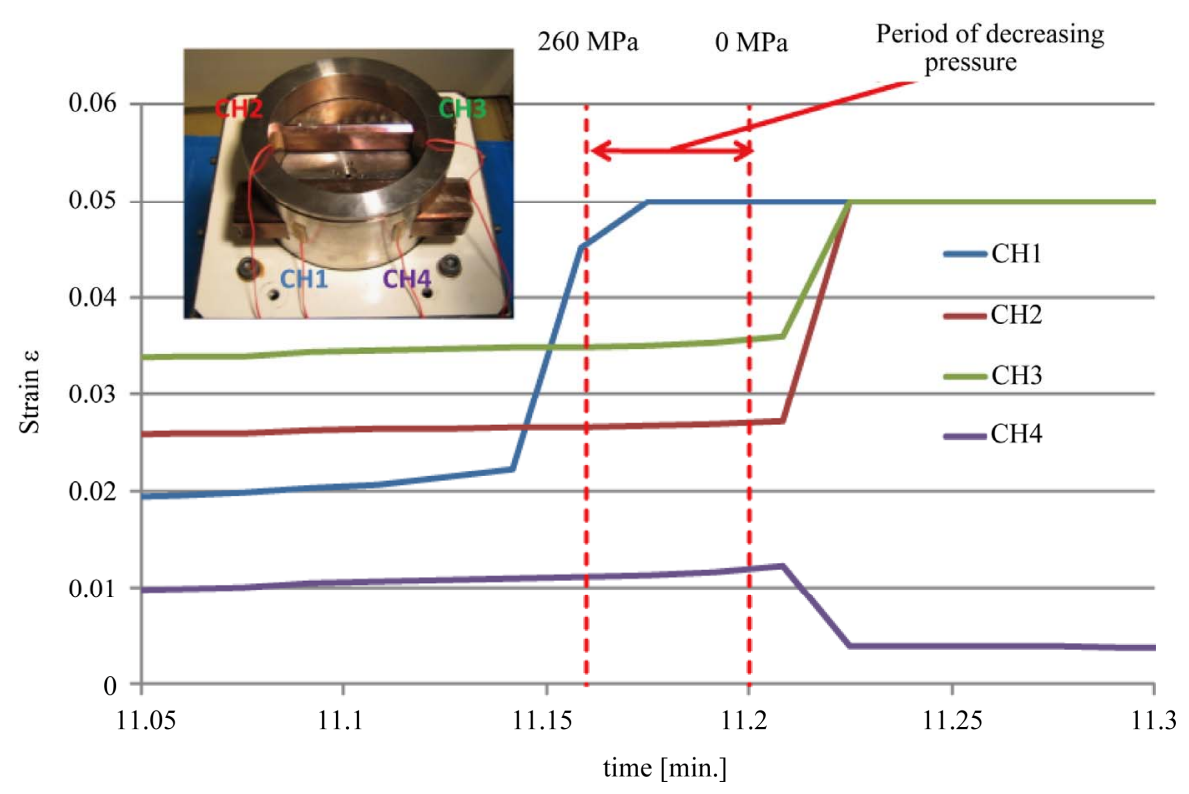

Figure 11. Change of strain during fracture propagation.

outer side when the sliding of the arm or clearance of the arm is considered. This analysis indicates the necessity of improvement of contacting conditions between arm and arm guide.

\section{Conclusions}

A double-wall high-pressure vessel in which the concept of the standby redundant system is introduced was proposed. The dimensions of the outer cover were decided by calculations that take the fracture mode into account, and fracture testing verifies the effectiveness of the outer cover. This research was financially supported by JST project "Development of Technology for Promoting
Food Quality”.

\section{References}

[1] Development of basic technologies available to Enhancing Value-Added to Food.

http://www.nico.or.jp/ create/gaiyou/index.html

[2] H. B. Haron, Y. Mutoh, Y. Otsuka and Y. Miyashita, "Selection of Stress Relief Grooves On Pressure Vessels for Food Processing," Proceedings of JSME Hokuriku Sinetsu Branch 48th General Conference and Lecture Meeting CD-ROM (No. 616), Ueda, 8 March 2011.

[3] M. G. Stewart and R. E. Melchers, "Probabilistic Risk Assessment of Engineering Systems,” Morikita Publishing Inc., Tokyo, 2003. 